1. Choudhury Y, et al. Attenuated adenosine-toinosine editing of microRNA-376a* promotes invasiveness of glioblastoma cells. J Clin Invest. 2012;122(11):4059-4076.

2. Pasquinelli AE. MicroRNAs and their targets: recognition, regulation and an emerging reciprocal relationship. Nat Rev Genet. 2012;13(4):271-282.

3. Iorio MV, Croce CM. microRNA involvement in human cancer. Carcinogenesis. 2012;33(6):1126-1133.

4. Levanon EY, et al. Systematic identification of abundant A-to-I editing sites in the human transcriptome. Nat Biotechnol. 2004;22(8):1001-1005

5. Kim DD, et al. Widespread RNA editing of embedded alu elements in the human transcriptome. Genome Res. 2004;14(9):1719-1725.

6. Nishikura K. Functions and regulation of RNA editing by ADAR deaminases. Annu Rev Biochem. 2010;79:321-349.

7. Prasanth KV, et al. Regulating gene expression through RNA nuclear retention. Cell. 2005;123(2):249-263.

8. Scadden AD, O'Connell MA. Cleavage of dsRNAs hyper-edited by ADARs occurs at preferred editing sites. Nucleic Acids Res. 2005;33(18):5954-5964.

9. Nie Y, Hammond GL, Yang JH. Double-stranded RNA deaminase ADAR1 increases host susceptibility to virus infection. J Virol. 2007;81(2):917-923.

10. Yang W, et al. Modulation of microRNA processing and expression through RNA editing by ADAR deaminases. Nat Struct Mol Biol. 2006;13(1):13-21.

11. Paz N, et al. Altered adenosine-to-inosine RNA editing in human cancer. Genome Res. 2007;
17(11):1586-1595.

12. Maas S, Patt S, Schrey M, Rich A. Underediting of glutamate receptor GluR-B mRNA in malignant gliomas. Proc Natl Acad Sci U S A. 2001;98(25):14687-14692.

13. Cenci C, et al. Down-regulation of RNA editing in pediatric astrocytomas: ADAR2 editing activity inhibits cell migration and proliferation. J Biol Chem. 2008;283(11):7251-7260.

14. Dunn GP, et al. Emerging insights into the molecular and cellular basis of glioblastoma. Genes Dev. 2012;26(8):756-784.

15. Singh D, et al. Transforming fusions of FGFR and TACC genes in human glioblastoma. Science. 2012;337(6099):1231-1235.

16. Solomon DA, et al. Mutational inactivation of STAG2 causes aneuploidy in human cancer. Science. 2011;333(6045):1039-1043.

17. Dominissini D, Moshitch-Moshkovitz S, Amariglio N, Rechavi G. Adenosine-to-inosine RNA editing meets cancer. Carcinogenesis. 2011;32(11):1569-1577.

18. Ishiuchi $\mathrm{S}$, et al. Blockage of $\mathrm{Ca}(2+)$-permeable AMPA receptors suppresses migration and induces apoptosis in human glioblastoma cells. Nat Med. 2002;8(9):971-978.

19. Luciano DJ, Mirsky H, Vendetti NJ, Maas S RNA editing of a miRNA precursor. RNA. 2004; 10(8):1174-1177.

20. Kawahara Y, Zinshteyn B, Sethupathy P, Iizasa H, Hatzigeorgiou AG, Nishikura K. Redirection of silencing targets by adenosine-to-inosine editing of miRNAs. Science. 2007;315(5815):1137-1140.
21. Kawahara Y, Zinshteyn B, Chendrimada TP, Shiekhattar R. Nishikura K. RNA editing of the microRNA-151 precursor blocks cleavage by the Dicer-TRBP complex. EMBO Rep. 2007;8(8):763-769.

22. Iizasa $H$, et al. Editing of Epstein-Barr virus-encoded BART6 microRNAs controls their dicer targeting and consequently affects viral latency. J Biol Chem. 2010;285(43):33358-33370.

23. Ekdahl Y, Farahani HS, Behm M, Lagergren J, Ohman M. A-to-I editing of microRNAs in the mammalian brain increases during development. Genome Res. 2012;22(8):1477-1487.

24. Alon S, et al. Systematic identification of edited microRNAs in the human brain. Genome Res. 2012;22(8):1533-1540.

25. Baylin SB, Jones PA. A decade of exploring the cancer epigenome - biological and translational implications. Nat Rev Cancer. 2011;11(10):726-734

26. Dawson MA, Kouzarides T. Cancer epigenetics: from mechanism to therapy. Cell. 2012;150(1):12-27.

27. Shih AH, Abdel-Wahab O, Patel JP, Levine RL. The role of mutations in epigenetic regulators in myeloid malignancies. Nat Rev Cancer. 2012;12(9):599-612.

28. Arrowsmith CH, Bountra C, Fish PV, Lee K, Schapira $\mathrm{M}$. Epigenetic protein families: a new frontier for drug discovery. Nat Rev Drug Discov. 2012;11(5):384-400.

29. He C. Grand challenge commentary: RNA epigenetics? Nat Chem Biol. 2010;6(12):863-865.

30. Wulff BE, Sakurai M, Nishikura K. Elucidating the inosinome: global approaches to adenosineto-inosine RNA editing. Nat Rev Genet. 2011; $12(2): 81-85$.

\title{
Lung capillaries raise the hypoxia alarm
}

\section{Jahar Bhattacharya}

Division of Pulmonary, Allergy and Critical Care, Department of Medicine, Department of Physiology and Cellular Biophysics, College of Physicians and Surgeons, Columbia University, New York, New York, USA.

\begin{abstract}
When ventilation is blocked, the lung can protect against the loss of blood oxygenation by activating localized arterial vasoconstriction, reducing blood flow to underventilated regions, and redirecting flow to better-ventilated alveoli. This phenomenon, hypoxic pulmonary vasoconstriction (HPV), preserves the overall efficiency of blood oxygenation, but the mechanism by which the hypoxic signal is transmitted to the smooth muscle that contracts the arterioles has remained largely a mystery. In this issue of the JCI, Wang et al. reveal that the endothelial lining of the hypoxic alveoli plays a key role in sensing hypoxia and transmitting the signal to initiate HPV.
\end{abstract}

The equilibrium between ventilation to a lung region and the region's blood supply establishes blood oxygenation, the critical physiological outcome that ensures oxygen delivery to tissues. Multiple lung diseases threaten this equilibrium, potentially increasing the risk of systemic hypoxia. Hypoxic venous blood returning to the lungs gets oxygenated as it flows through alveolar capillaries, where oxygen diffuses

Conflict of interest: The author has declared that no conflict of interest exists.

Citation for this article: J Clin Invest. 2012; 122(11):3845-3847. doi:10.1172/JCI65623. into it from alveoli. Diseases that impair alveolar ventilation also impair blood oxygenation. This occurs, for example, in lung injury in which alveoli are inundated with plasma exudate and inflammatory cells and can therefore no longer be ventilated. Hypoxic blood streaming from diseased regions mixes with oxygenated blood streaming from well-ventilated regions. The admixture of the hypoxic streams potentially causes hypoxemia of the entire pulmonary outflow destined for systemic vascular beds. The lung protects against systemic hypoxemia by activating localized arterial vasoconstriction, which effectively turns off blood flow to underventilated regions, redirecting flow to better-ventilated alveoli and thereby restricting the formation of hypoxic streams. This phenomenon, commonly called hypoxic pulmonary vasoconstriction (HPV), preserves the overall efficacy of blood oxygenation. As Sylvester et al. discuss in a recent review (1), HPV has been long recognized, but is not entirely understood at the mechanistic level.

\section{Signal sensing for HPV}

In moderate hypoxia, defined as a fall in the oxygen tension of alveolar gas to $30-50$ $\mathrm{mmHg}, \mathrm{HPV}$ is seen within minutes, which indicates that the hypoxic signal rapidly traverses the spatial separation between the alveoli and the upstream arteriolar segment, the site of vasoconstriction. Since smooth muscle contraction is required for HPV, the bulk of the research in the field has focused on mechanisms of smooth muscle contractility. In general, these mechanisms are based on the notion that hypoxia of pulmonary arterial smooth 


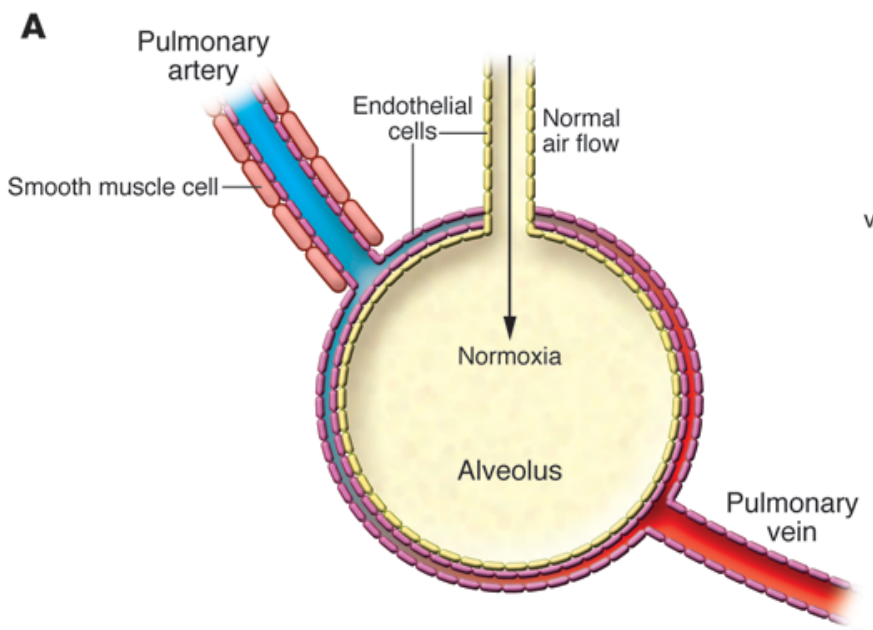

B

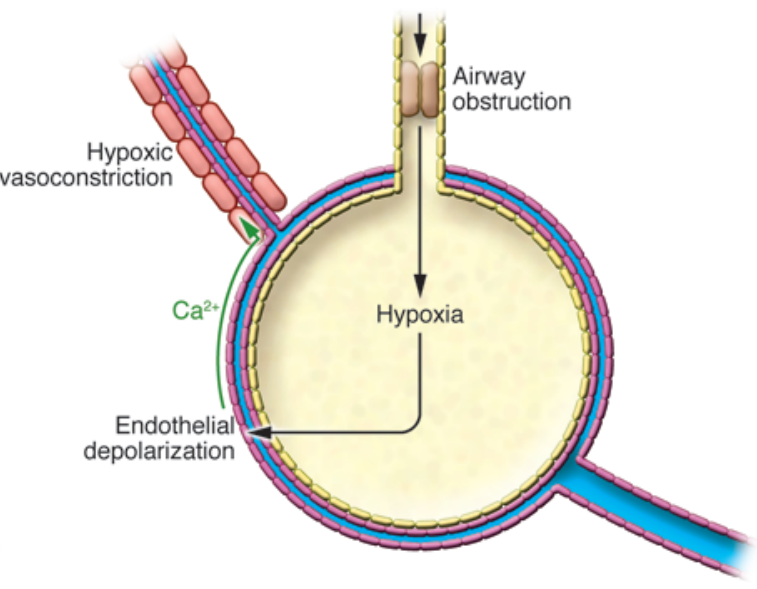

\section{Figure 1}

Essential features of the capillary mechanism of HPV. Mixed venous blood flows in through the pulmonary artery that branches to arterioles and then alveolar capillaries. The vascular smooth muscle investment ends at the level of the arterioles. The capillaries surround the air-filled alveolus. (A) In the normally ventilated alveolus, the hypoxic blood (blue) becomes oxygenated (red) and leaves the lung through the pulmonary veins. (B) Blockade of ventilation by airway disease causes alveolar hypoxia. The capillary endothelium is depolarized. The resulting increase of endothelial $\mathrm{Ca}^{2+}$ is communicated through endothelial gap junctions to smooth muscle surrounding upstream arterioles. Smooth muscle activation causes arteriolar constriction, restricting blood flow in the underventilated region.

muscle decreases mitochondrial oxidative phosphorylation, leading to contractilityinducing events such as $\mathrm{K}^{+}$channel closure and cytosolic $\mathrm{Ca}^{2+}$ increase (1). Since these mechanisms are evidently activated after the smooth muscle has already been exposed to hypoxia, it remains unclear how hypoxia itself, or a signal induced by hypoxia, is conveyed from underventilated, hypoxic alveoli to upstream arterioles.

One possibility is that the signal passes by direct diffusion. Weibel proposed that oxygen diffusion could occur from alveoli to all surrounding tissues (2). Jameson confirmed this prediction, showing that when alveoli are hyperoxic, as during pure oxygen breathing, oxygen diffuses to pulmonary arteries (3). However, the picture is different for normoxic alveoli. In their classic paper, Conhaim and Staub determined the extent to which precapillary blood undergoes oxygenation at different levels of alveolar oxygen tension, thereby reflecting the extent of oxygen diffusion from alveoli to the arteriolar segment (4). Although their findings for high oxygen breathing agree with Jameson's interpretation that alveolar hyperoxia is capable of driving oxygen diffusion to the arterioles, their critical finding was that the diffusion is limited when alveoli are normoxic. Hence, it is unlikely that significant diffusion to the arterioles occurs when the alveoli are hypoxic, when the alveolus-arteriole oxygen gradient is probably even smaller than in normoxia, leaving open the question of how the arteriolar smooth muscle senses alveolar hypoxia.

\section{A new model for signal sensing}

In this issue of the JCI, the paper by Wang et al. from the Kuebler group reports a remarkably elegant solution to this longstanding puzzle (5). The authors found that the alveolar capillary endothelium plays a primary role in sensing alveolar hypoxia as well as in providing a conduit for signals that alert upstream arterioles to the hypoxia. The hypoxia sensing takes place in alveolar capillaries as a consequence of hypoxia-induced endothelial $\mathrm{K}^{+}$ channel closure, which leads to endothelial membrane depolarization and $\mathrm{Ca}^{2+}$ entry. The conduit for signal transmission is provided by endothelial gap junctional communication, in which the $\mathrm{Ca}^{2+}$ increase passes from the alveolar to the upstream arteriolar endothelium. Arteriolar constriction ensues as the $\mathrm{Ca}^{2+}$-activated arteriolar endothelium induces paracrine stimulation of the surrounding smooth muscle (Figure 1).

The beauty of this proposal is that it is completely out of the box. For the first time, it places the first-alert warning system for hypoxia at the primary site of blood oxygenation, namely the alveoli. The capillary endothelium is clearly well posi- tioned in this regard, since the endothelial membrane lies immediately adjacent to the alveolar epithelial membrane. This double membrane is in places only fractions of a micron thick and is therefore exquisitely engineered for rapid oxygen diffusion. These features ensure an agile hypoxiasensing system that would probably not be surpassed were the sensing sites to lie further from the alveoli.

This proposal from Wang et al. also brings into focus the increasing understanding that the intercellular connectivity of lung microvessels determines pathophysiological responses in the lung. The lung microvascular endothelium richly expresses several connexins, the constitutive components of intercellular gap junctional channels. The lung's endothelial lining, formed by gap junction-connected endothelial cells, is in fact a syncytium that can rapidly transmit diffusible signals across relatively large distances of the vascular bed. Previous work has shown that this syncytium is important for the spread of signals that amplify the lung's immune response to injurious agents (6). Now, Wang et al. show that the endothelial syncytium transmits hypoxia-generated signals from the alveoli to the arterioles (5). In this case, the endothelial syncytium provides a high-speed throughway that rapidly conveys early warning signals to activate the defensive HPV response. 


\section{An imperfect system?}

When could this elegant hypoxia-alert mechanism fail? Wang et al. suggest one potential scenario, namely during severe hypoxia. They found that when alveolar hypoxia is sufficiently intense as to decrease the blood oxygen tension to well below $30 \mathrm{mmHg}$, the endothelial depolarization that is critical to the hypoxia alert does not occur. Consequently, there is no increase in endothelial $\mathrm{Ca}^{2+}$ and, presumably, no transmission of any $\mathrm{Ca}^{2+}$-dependent warning signals to arteriolar vessels through endothelial gap junctions. A similar failure of capillary signaling could occur in conditions such as lung injury if there is endothelial damage, causing loss of endothelial gap junctional communication. Acute lung inflammation decreases lung expression of connexin 40 (7), the same connexin that alerts arterioles to alveolar hypoxia (5). In fact, the finding that HPV is abrogated in connexin 40-knockout mice (5) suggests that in lung injury, loss of signaling through endothelial gap junctions might contribute to HPV failure, and hence hypoxemia severity, in this condition.

The larger significance of these findings in lung vascular biology might relate to other capillary responses that are reported to occur after activation of voltage-depen- dent $\mathrm{Ca}^{2+}$ channels. Stoppage of lung blood flow causes endothelial membrane depolarization in lung capillaries, leading to endothelial $\mathrm{Ca}^{2+}$ entry through the voltagedependent $\mathrm{Ca}^{2+}$ channel $\alpha_{1 \mathrm{G}}$ - the same channel studied by Wang et al. - to cause release of reactive oxygen species through NADPH oxidase induction (8). The PAR-1 activator thrombin also induces endothelial $\mathrm{Ca}^{2+}$ entry through the $\alpha_{1 \mathrm{G}}$ channel to activate endothelial expression of the leukocyte adhesion receptor P-selectin in alveolar capillaries (9). Taking these diverse findings together, it would seem that voltage-activated $\mathrm{Ca}^{2+}$ entry forms a fulcrum for multiple lung vascular phenomena that could potentially coalesce into a pathogenic process. Clearly, more needs to be known as to how voltage-dependent $\mathrm{Ca}^{2+}$ entry in lung endothelium co-opts gap junctional communication to promote vascular diseases of the lung. Nevertheless, the mechanisms of hypoxia sensing now identified by Wang et al. open up possibilities for targeting voltage-gated channels and gap junctional proteins in the therapy of diseases in which loss of the protection by HPV is a known adverse factor.

\section{Acknowledgments}

The author acknowledges Sunita Bhattacharya and Mallar Bhattacharya for reading the manuscript. J. Bhattacharya is supported by NIH grants HL36024 and HL64896.

Address correspondence to: Jahar Bhattacharya, Columbia University College of Physicians and Surgeons, Lung Biology Laboratory, Department of Medicine, 630 West 168th Street, BB 8-812, New York, New York 10032, USA. Phone: 212.305.7093; Fax: 212.305.6701; E-mail: jb39@columbia.edu.

1. Sylvester JT, Shimoda LA, Aaronson PI, Ward JP. Hypoxic pulmonary vasoconstriction. Physiol Rev. 2012;92(1):367-520.

2. Weibel E. [Blood vessel anastomoses in the human lungs]. Z Zellforsch Mikrosk Anat. 1959;50:653-692.

3. Jameson AG. Diffusion of gases from alveolus to precapillary arteries. Science. 1963;139(3557):826-828.

4. Conhaim RL, Staub NC. Reflection spectrophotometric measurement of $\mathrm{O} 2$ uptake in pulmonary arterioles of cats. J Appl Physiol. 1980;48(5):848-856.

5 . Wang L, et al. Hypoxic pulmonary vasoconstriction requires connexin 40-mediated endothelial signal conduction. J Clin Invest. 2012;122(11):4218-4230.

6. Parthasarathi K, et al. Connexin 43 mediates spread of $\mathrm{Ca} 2+$-dependent proinflammatory responses in lung capillaries. JClin Invest. 2006;116(8):2193-2200.

7. Rignault S, Haefliger JA, Waeber B, Liaudet L, Feihl F. Acute inflammation decreases the expression of connexin 40 in mouse lung. Shock. 2007;28(1):78-85.

8. Wei Z, Manevich Y, Al-Mehdi AB, Chatterjee $\mathrm{S}$, Fisher AB. Ca2+ flux through voltage-gated channels with flow cessation in pulmonary microvascular endothelial cells. Microcirculation. 2004;11(6):517-526.

9. Zhou C, et al. Alpha1G T-type calcium channel selectively regulates P-selectin surface expression in pulmonary capillary endothelium. Am J Physiol Lung Cell Mol Physiol. 2010;299(1):L86-L97.

\title{
The bitter taste of infection
}

\author{
Alice Prince \\ Department of Pediatrics, Columbia University, New York, New York, USA.
}

\begin{abstract}
The human innate immune response to pathogens is complex, and it has been difficult to establish the contribution of epithelial signaling in the prevention of upper respiratory tract infection. The prevalence of chronic sinusitis in the absence of systemic immune defects indicates that there may be local defects in innate immunity associated with such mucosal infections. In this issue of the JCI, Cohen and colleagues investigate the role of the bitter taste receptors in airway epithelial cells, and find that these are critical to sensing the presence of invading pathogens.
\end{abstract}

The participation of respiratory mucosal epithelial cells in innate immune defense has been increasingly appreciated. Not only do airway cells express the full complement

Conflict of interest: The author has declared that no conflict of interest exists.

Citation for this article: J Clin Invest. 2012; 122(11):3847-3849. doi:10.1172/JCI66182. of pattern recognition receptors and corresponding adaptor proteins to signal the recruitment of professional immune cells in response to perceived infection, they also participate directly in pathogen eradication. Mucociliary clearance is activated in response to bacterial components, and bacterial killing is mediated through epithelial production of NO and antimicrobial pep- tides. Although major defects in ciliary function (e.g., Kartagener syndrome) are clearly associated with increased respiratory infection rates, more subtle epithelial abnormalities that might be important in susceptibility to common conditions such as chronic sinus infection have not been fully characterized. Mutations in cystic fibrosis transmembrane conductance regulator (CFTR) that do not cause cystic fibrosis have been associated with chronic rhinosinusitis, although the specific pathogenetic mechanisms involved have not been determined (1). Given the complexity of the human innate immune response to pathogens, it has been difficult to establish the contribution of epithelial signaling in the prevention of upper respiratory tract infection. Nonetheless, given the 